CRYSTALLOGRAPHIC COMMUNICATIONS

ISSN 2056-9890

Received 4 March 2015

Accepted 3 April 2015

Edited by H. Ishida, Okayama University, Japan

Keywords: crystal structure; packing polymorphism; 2-nitrobenzyl ester; $\pi-\pi$ interactions; $\mathrm{C}-\mathrm{H} \cdots \mathrm{O}$ interactions

CCDC references: 967703; 967704 Supporting information: this article has supporting information at journals.iucr.org/e

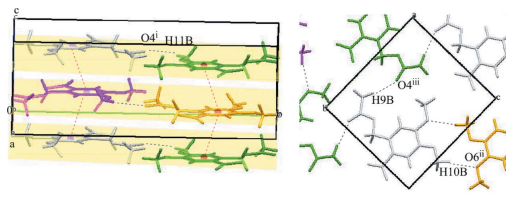

OPEN $\odot$ ACCESS

\section{Packing polymorphism in the crystal structure of 4,5-dimethoxy-2-nitrobenzyl acetate}

\author{
Noriko Chikaraishi Kasuga, ${ }^{\mathrm{a}}$ Yusuke Saito, ${ }^{\mathrm{a}}$ Hiroyasu Sato ${ }^{\mathrm{b}}$ and Kazuo Yamaguchi ${ }^{\mathrm{a} *}$ \\ aDepartment of Materials Science, Faculty of Science, Kanagawa University, Tsuchiya, Hiratsuka, Kanagawa 259-1293, \\ Japan, and ${ }^{\mathbf{b}}$ Rigaku Corporation 3-9-12 Matsubara-cho, Akishima, Tokyo 196-8666, Japan. *Correspondence e-mail: \\ kazu@kanagawa-u.ac.jp
}

The title compound, $\mathrm{C}_{11} \mathrm{H}_{13} \mathrm{NO}_{6}$, shows two polymorphs, orange and yellow forms, both of which crystallize in the space group $P 2_{1} / c$. The molecular structures in the two polymorphs are essentially similar and adopt a planar structure, the maximum deviations for the non-H atoms being 0.1836 (13) and 0.1276 (13) $\AA$, respectively, for the orange and yellow forms. In the orange crystal, molecules are linked by an intermolecular $\mathrm{C}-\mathrm{H} \cdots \mathrm{O}$ interaction into a helical chain along the $b$-axis direction. The chains are stacked along the $c$ axis through a $\pi-\pi$ interaction [centroid-centroid distance $=3.6087$ (11) $\AA$ ], forming a layer parallel to the $b c$ plane. In the yellow crystal, molecules are connected through $\mathrm{C}-\mathrm{H} \cdots \mathrm{O}$ interactions into a sheet structure parallel to $(\overline{3} 02)$. No significant $\pi-\pi$ interaction is observed. The unit-cell volume of the orange crystal is larger than that of the yellow one, and this accounts for the predominant growth of the yellow crystal.

\section{Chemical context}

Polymorphism is of interest in crystallization, phase transition, material synthesis and the pharmaceutical industry because differences in the crystal packing and/or conformation of compounds with the same formula can change the chemical and physical properties, including solubility, bioavailability and so forth (Moulton \& Zaworotko, 2001; Matsuo \& Matsuoka, 2007; Yu, 2010). We have been investigating silane coupling agents and thiols with distal functional groups protected by photolabile 2-nitrobenzyl groups (Edagawa et al., 2012). During the course of photoremoval studies of these materials, we found that the simple ester, 4,5-dimethoxy-2nitrobenzyl acetate, which releases acetic acid on photo-irradiation, forms two different types of crystals, orange rods and yellow needles. Here, we report the crystal structures of these two polymorphs of the title compound.<smiles>COc1cc(COC(C)=O)c([N+](=O)[O-])cc1OC</smiles>

\section{Structural commentary}

The molecular structures of the two crystals are approximately planar and almost identical, as shown in Fig. 1. The $\mathrm{C} 2-\mathrm{C} 1-$ $\mathrm{C} 7-\mathrm{O} 3, \mathrm{C} 9-\mathrm{C} 8-\mathrm{O} 3-\mathrm{C} 7, \mathrm{C} 5-\mathrm{C} 4-\mathrm{O} 5-\mathrm{C} 10$ and $\mathrm{C} 4-$ 


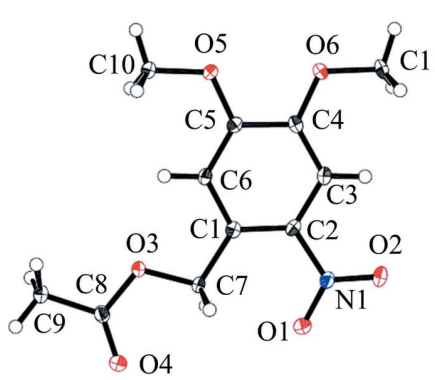

orange

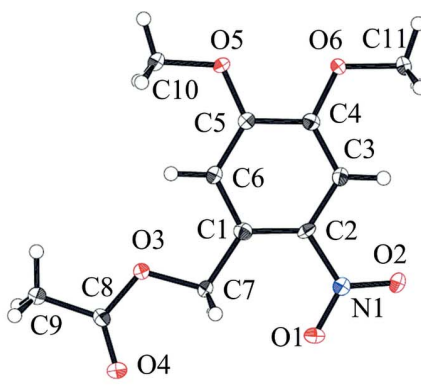

yellow
Figure 1

The molecular structures of the title compound polymorphs, with atom labelling. Displacement ellipsoids are drawn at the $50 \%$ probability level.

C5-O6-C11 torsion angles in the two crystals are approximately $180^{\circ}$. The dihedral angles between the benzene ring $(\mathrm{C} 1-\mathrm{C} 6)$ and the nitro group $(\mathrm{O} 1 / \mathrm{N} 1 / \mathrm{O} 2)$ are $9.54(11)$ and $4.15(7)^{\circ}$ for the orange and yellow polymorphs, respectively.

\section{Supramolecular features}

Although the two crystals crystallize in the same space group $\left(P 2_{1} / c\right)$ with $Z^{\prime}=1$, their packing modes are different. In the orange crystal, the molecules are connected by an intermolecular $\mathrm{C}-\mathrm{H} \cdots \mathrm{O}$ interaction $\left[\mathrm{C} 11-\mathrm{H} 11 B \cdots \mathrm{O} 4^{\mathrm{i}}\right.$; symmetry code: (i) $1-x,-\frac{1}{2}+y, \frac{3}{2}-z$; Table 1 ] between the methoxy group and the carbonyl group, forming a helical chain along the $b$ axis as shown in Fig. 2, left. In addition, a $\pi-\pi$ interaction between the benzene rings with a centroidcentroid distance of 3.6087 (11) $\AA$ links the chains to be stacked along the $c$ axis. In the yellow crystal, the molecules located in the plane perpendicular to the $a c$ plane are connected by $\mathrm{C}-\mathrm{H} \cdots \mathrm{O}$ interactions (Table 2) between methoxy groups $\left[\mathrm{C} 10-\mathrm{H} 10 B \cdots \mathrm{O} 6^{\mathrm{ii}}\right.$; symmetry code: (ii) $1-x$, $1-y, 2-z]$ and between acetyl groups $\left[\mathrm{C} 9-\mathrm{H} 9 B \cdots \mathrm{O} 4^{\text {iiii }}\right.$; symmetry code: (iii) $\left.-x,-\frac{1}{2}+y, \frac{1}{2}-z\right]$, forming a sheet structure parallel to ( $\overline{3} 02)$ (Fig. 2, right).
Table 1

Hydrogen-bond geometry $\left(\AA,^{\circ}\right)$ for orange.

\begin{tabular}{|c|c|c|c|c|}
\hline$D-\mathrm{H} \cdots A$ & $D-\mathrm{H}$ & $\mathrm{H} \cdots A$ & $D \cdots A$ & $D-\mathrm{H} \cdots A$ \\
\hline $\mathrm{C} 11-\mathrm{H} 11 B \cdots \mathrm{O} 4^{\mathrm{i}}$ & 0.98 & 2.50 & 3.369 (2) & 147 \\
\hline
\end{tabular}

Symmetry code: (i) $-x+1, y-\frac{1}{2},-z+\frac{3}{2}$.

Table 2

Hydrogen-bond geometry $\left(\AA{ }^{\circ}\right)$ for yellow.

\begin{tabular}{lllll}
\hline$D-\mathrm{H} \cdots A$ & $D-\mathrm{H}$ & $\mathrm{H} \cdots A$ & $D \cdots A$ & $D-\mathrm{H} \cdots A$ \\
\hline $\mathrm{C} 9-\mathrm{H} 9 B \cdots \mathrm{O} 4^{\mathrm{iii}}$ & 0.98 & 2.40 & $3.375(2)$ & 174 \\
$\mathrm{C} 10-\mathrm{H} 10 B \cdots \mathrm{O} 6^{\mathrm{ii}}$ & 0.98 & 2.51 & $3.472(2)$ & 169 \\
\hline
\end{tabular}

Symmetry codes: (ii) $-x+1,-y+1,-z+2$; (iii) $-x, y-\frac{1}{2},-z+\frac{1}{2}$.

In the orange crystal, the molecules are stacked in columnar structures via $\pi-\pi$ interactions along the $c$ axis (Fig. 3, left). In contrast, no $\pi-\pi$ interactions are observed in the yellow crystal. The molecules are therefore terraced along the diagonal line of the $a$ and $c$ axes as shown in Fig. 3, right. As a result of these packing differences, the volume of the unit cell of the orange crystal is larger than that of the yellow one, i.e., the orange crystal contains slightly more void space than the yellow one. This would account for the predominant growth of the yellow crystals.

\section{Synthesis and crystallization}

4,5-Dimethoxy-2-nitrobenzyl alcohol $(0.714 \mathrm{~g}, 3.35 \mathrm{mmol})$, acetic anhydride $(0.63 \mathrm{ml}, 6.66 \mathrm{mmol}), \mathrm{Et}_{3} \mathrm{~N}(1 \mathrm{ml})$ and $\mathrm{CH}_{2} \mathrm{Cl}_{2}(20 \mathrm{ml})$ were placed in a $100 \mathrm{~mL}$ flask, and the mixture was stirred at ambient temperature overnight. The mixture was extracted with $\mathrm{CH}_{2} \mathrm{Cl}_{2}(20 \mathrm{ml} \times 3)$, washed with brine, dried over $\mathrm{MgSO}_{4}$, and evaporated to give a yellow solid $(0.773 \mathrm{~g}, 90 \%$ yield $)$. The solid was crystallized by slow evaporation from a mixed solution of ethyl acetate and hexane

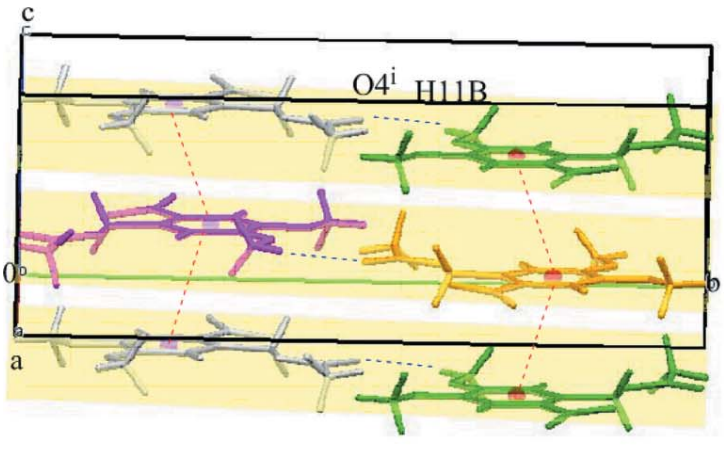

orange

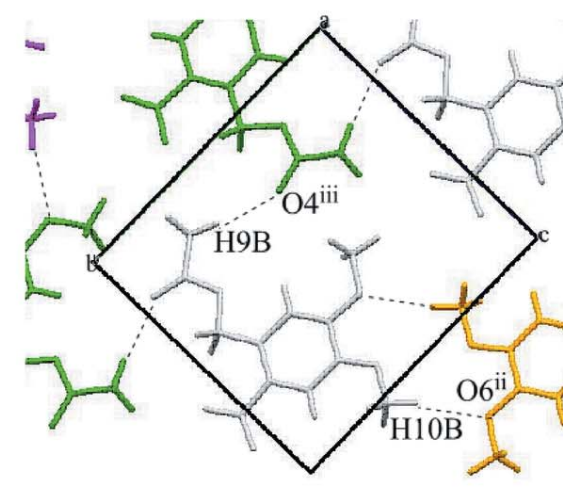

yellow

Figure 2

Intermolecular $\mathrm{C}-\mathrm{H} \cdots \mathrm{O}$ (black dashed lines) and $\pi-\pi$ (red dashed lines) interactions in the orange crystal (left), and intermolecular $\mathrm{C}-\mathrm{H} \cdots \mathrm{O}$ interactions (black dashed lines) between methoxy groups and between acetyl groups in the yellow crystal (right). [Symmetry codes: (i) $1-x,-\frac{1}{2}+y$, $\frac{3}{2}-z$; (ii) $1-x, 1-y, 2-z$; (iii) $-x,-\frac{1}{2}+y, \frac{1}{2}-z$.] 


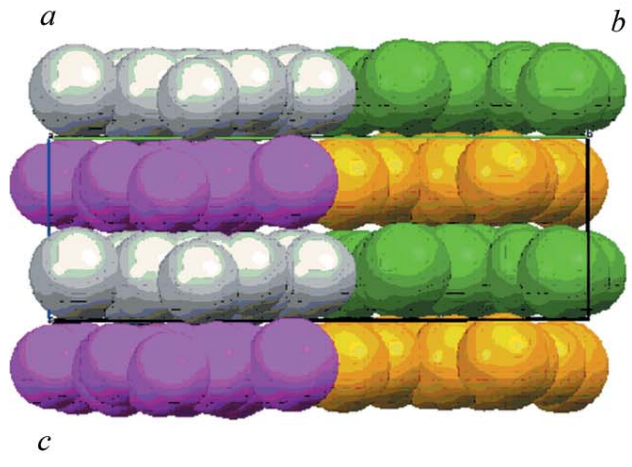

orange

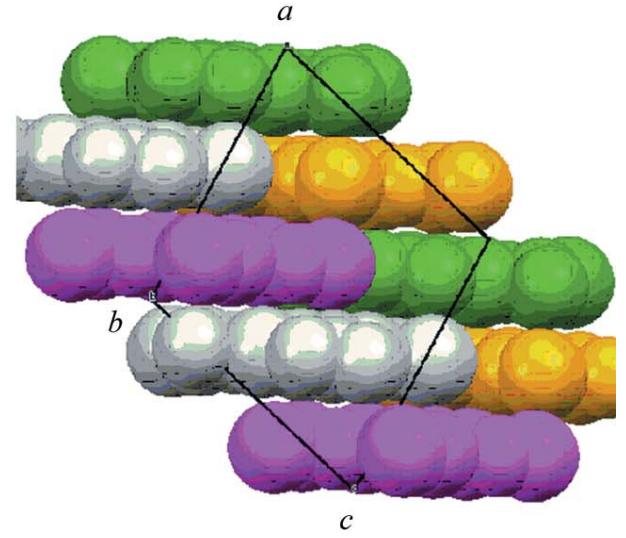

yellow

Figure 3

Side views of space-filling models of molecular packing of the orange (left) and yellow (right) crystals.

(1:1). Orange crystals were occasionally obtained in small amounts, but the yellow crystals grew predominantly.

\section{Refinement}

Crystal data, data collection and structure refinement details are summarized in Table 3. All $\mathrm{H}$ atoms were located geometrically and refined using a riding model, with $\mathrm{C}-\mathrm{H}=$ $0.99 \AA$ and $U_{\text {iso }}(\mathrm{H})=1.2 U_{\text {eq }}(\mathrm{C})$ for methylene $\mathrm{H}$ atoms, $\mathrm{C}-\mathrm{H}$ $=0.95 \AA$ and $U_{\text {iso }}(\mathrm{H})=1.2 U_{\text {eq }}(\mathrm{C})$ for aromatic $\mathrm{H}$ atoms, and $\mathrm{C}-\mathrm{H}=0.98 \AA$ and $U_{\text {iso }}(\mathrm{H})=1.5 U_{\text {eq }}(\mathrm{C})$ for methyl $\mathrm{H}$ atoms.

\section{Acknowledgements}

We thank Kanagawa University for the general support of our studies.

\section{References}

Burla, M. C., Caliandro, R., Camalli, M., Carrozzini, B., Cascarano, G. L., De Caro, L., Giacovazzo, C., Polidori, G. \& Spagna, R. (2005). J. Appl. Cryst. 38, 381-388.

Edagawa, Y., Nakanishi, J., Yamaguchi, K. \& Takeda, N. (2012). Colloids Surf. B Biointerfaces, 99, 20-26.

Macrae, C. F., Bruno, I. J., Chisholm, J. A., Edgington, P. R., McCabe, P., Pidcock, E., Rodriguez-Monge, L., Taylor, R., van de Streek, J. \& Wood, P. A. (2008). J. Appl. Cryst. 41, 466-470.

Table 3

Experimental details.

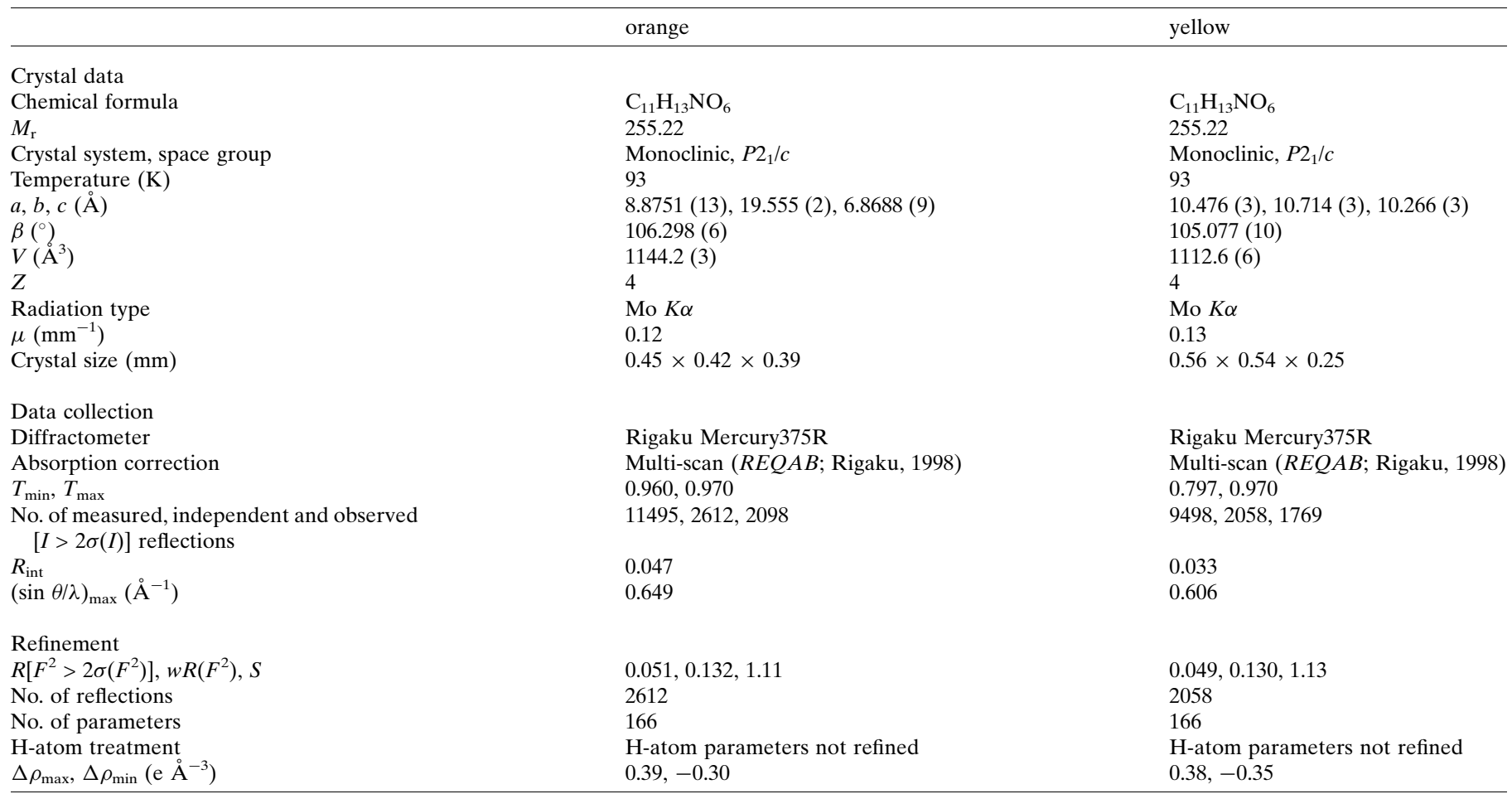

Computer programs: CrystalClear-SM Expert (Rigaku, 2011), SIR2004 (Burla et al., 2005), SHELXL97 (Sheldrick, 2008), Mercury (Macrae et al., 2008), Yadokari-XG (Wakita, 2001). 
Matsuo, K. \& Matsuoka, M. (2007). Cryst. Growth Des. 7, 411-415.

Moulton, B. \& Zaworotko, M. J. (2001). Chem. Rev. 101, 1629-1658.

Rigaku (1998). REQAB. Rigaku Corporation, Tokyo, Japan.

Rigaku (2011). CrystalClear-SM Expert. Rigaku Corporation, Tokyo, Japan.
Sheldrick, G. M. (2008). Acta Cryst. A64, 112-122.

Wakita, K. (2001). Yadokari-XG. http://www.hat.hi-ho.jp/k-wakita/ yadokari

Yu, L. (2010). Acc. Chem. Res. 43, 1257-1266. 


\section{supporting information}

Acta Cryst. (2015). E71, 483-486 [https://doi.org/10.1107/S2056989015006714]

Packing polymorphism in the crystal structure of 4,5-dimethoxy-2-nitrobenzyl acetate

\section{Noriko Chikaraishi Kasuga, Yusuke Saito, Hiroyasu Sato and Kazuo Yamaguchi}

Computing details

For both compounds, data collection: CrystalClear-SM Expert (Rigaku, 2011). Cell refinement: CrystalClear-SM Expert (Rigaku, 2011) for orange; CrystalClear-SM Expert for yellow. Data reduction: CrystalClear-SM Expert (Rigaku, 2011) for orange; CrystalClear-SM Expert for yellow. For both compounds, program(s) used to solve structure: SIR2004 (Burla et al., 2005); program(s) used to refine structure: SHELXL97 (Sheldrick, 2008); molecular graphics: Mercury (Macrae et al., 2008); software used to prepare material for publication: Yadokari-XG (Wakita, 2001).

(orange) 4,5-Dimethoxy-2-nitrobenzyl acetate

Crystal data

$\mathrm{C}_{11} \mathrm{H}_{13} \mathrm{NO}_{6}$

$M_{r}=255.22$

Monoclinic, $P 2_{1} / c$

Hall symbol: -P 2ybc

$a=8.8751$ (13) $\AA$

$b=19.555(2) \AA$

$c=6.8688(9) \AA$

$\beta=106.298(6)^{\circ}$

$V=1144.2(3) \AA^{3}$

$Z=4$

\section{Data collection}

Rigaku Mercury375R (2x2 bin mode) diffractometer

Radiation source: fine-focus sealed tube Graphite monochromator

Detector resolution: 13.6612 pixels $\mathrm{mm}^{-1}$ profile data from $\omega$-scans

Absorption correction: multi-scan

(REQAB; Rigaku, 1998)

$T_{\min }=0.960, T_{\max }=0.970$

\section{Refinement}

Refinement on $F^{2}$

Least-squares matrix: full

$R\left[F^{2}>2 \sigma\left(F^{2}\right)\right]=0.051$

$w R\left(F^{2}\right)=0.132$

$S=1.11$

2612 reflections

166 parameters
$F(000)=536$

$D_{\mathrm{x}}=1.48 \mathrm{Mg} \mathrm{m}^{-3}$

Mo $K \alpha$ radiation, $\lambda=0.71069 \AA$

Cell parameters from 2655 reflections

$\theta=3.1-27.5^{\circ}$

$\mu=0.12 \mathrm{~mm}^{-1}$

$T=93 \mathrm{~K}$

Platelet, orange

$0.45 \times 0.42 \times 0.39 \mathrm{~mm}$

11495 measured reflections

2612 independent reflections

2098 reflections with $I>2 \sigma(I)$

$R_{\text {int }}=0.047$

$\theta_{\max }=27.5^{\circ}, \theta_{\min }=3.2^{\circ}$

$h=-11 \rightarrow 11$

$k=-25 \rightarrow 25$

$l=-8 \rightarrow 8$

0 restraints

Primary atom site location: structure-invariant direct methods

Secondary atom site location: difference Fourier map

Hydrogen site location: inferred from neighbouring sites 
$\mathrm{H}$-atom parameters not refined

$w=1 /\left[\sigma^{2}\left(F_{\mathrm{o}}^{2}\right)+(0.0597 P)^{2}+0.5862 P\right]$

where $P=\left(F_{\mathrm{o}}^{2}+2 F_{\mathrm{c}}{ }^{2}\right) / 3$
$(\Delta / \sigma)_{\max }<0.001$
$\Delta \rho_{\max }=0.39 \mathrm{e} \AA^{-3}$
$\Delta \rho_{\min }=-0.30 \mathrm{e} \AA^{-3}$

Special details

Geometry. All e.s.d.'s (except the e.s.d. in the dihedral angle between two 1.s. planes) are estimated using the full covariance matrix. The cell e.s.d.'s are taken into account individually in the estimation of e.s.d.'s in distances, angles and torsion angles; correlations between e.s.d.'s in cell parameters are only used when they are defined by crystal symmetry. An approximate (isotropic) treatment of cell e.s.d.'s is used for estimating e.s.d.'s involving 1.s. planes.

Refinement. Refinement of $F^{2}$ against ALL reflections. The weighted $R$-factor $w R$ and goodness of fit $S$ are based on $F^{2}$, conventional $R$-factors $R$ are based on $F$, with $F$ set to zero for negative $F^{2}$. The threshold expression of $F^{2}>\sigma\left(F^{2}\right)$ is used only for calculating $R$-factors(gt) etc. and is not relevant to the choice of reflections for refinement. $R$-factors based on $F^{2}$ are statistically about twice as large as those based on $F$, and $R$ - factors based on ALL data will be even larger.

Fractional atomic coordinates and isotropic or equivalent isotropic displacement parameters $\left(\hat{A}^{2}\right)$

\begin{tabular}{|c|c|c|c|c|}
\hline & $x$ & $y$ & $z$ & $U_{\text {iso }} * / U_{\text {eq }}$ \\
\hline $\mathrm{C} 1$ & $0.19328(19)$ & $0.29434(8)$ & $0.7664(2)$ & 0.0124 \\
\hline $\mathrm{C} 2$ & 0.04911 (19) & $0.26435(9)$ & $0.7614(3)$ & $0.0139(3)$ \\
\hline $\mathrm{C} 3$ & $0.02606(19)$ & $0.19342(8)$ & $0.7577(3)$ & 0.0140 \\
\hline $\mathrm{H} 3$ & -0.0739 & 0.1752 & 0.7541 & $0.017^{*}$ \\
\hline $\mathrm{C} 4$ & $0.1487(2)$ & $0.15013(8)$ & 0.7593 & 0.0139 \\
\hline $\mathrm{C} 5$ & 0.29755 (19) & $0.17865(8)$ & $0.7678(2)$ & 0.0125 \\
\hline C6 & 0.31701 (19) & $0.24911(8)$ & $0.7700(2)$ & 0.0128 \\
\hline H6 & 0.4171 & 0.2673 & 0.7740 & $0.015^{*}$ \\
\hline $\mathrm{C} 7$ & $0.22141(19)$ & $0.37064(8)$ & 0.7674 & $0.0138(3)$ \\
\hline H7A & 0.1502 & 0.3919 & 0.6450 & $0.017^{*}$ \\
\hline H7B & 0.2010 & 0.3916 & 0.8887 & $0.017^{*}$ \\
\hline $\mathrm{C} 8$ & $0.4225(2)$ & $0.44786(9)$ & 0.7575 & $0.0155(4)$ \\
\hline C9 & $0.5882(2)$ & $0.45412(9)$ & 0.7490 & 0.0218 \\
\hline H9A & 0.5938 & 0.4425 & 0.6124 & $0.033^{*}$ \\
\hline H9B & 0.6550 & 0.4227 & 0.8475 & $0.033^{*}$ \\
\hline $\mathrm{H} 9 \mathrm{C}$ & 0.6247 & 0.5012 & 0.7816 & $0.033^{*}$ \\
\hline $\mathrm{C} 10$ & $-0.0084(2)$ & $0.05009(9)$ & $0.7387(3)$ & $0.0200(4)$ \\
\hline $\mathrm{H} 10 \mathrm{~A}$ & -0.0436 & 0.0632 & 0.8565 & $0.030 *$ \\
\hline $\mathrm{H} 10 \mathrm{~B}$ & 0.0007 & 0.0002 & 0.7344 & $0.030 *$ \\
\hline $\mathrm{H} 10 \mathrm{C}$ & -0.0848 & 0.0660 & 0.6144 & $0.030^{*}$ \\
\hline $\mathrm{C} 11$ & $0.5657(2)$ & $0.15867(9)$ & $0.7809(3)$ & $0.0187(4)$ \\
\hline $\mathrm{H} 11 \mathrm{~A}$ & 0.5598 & 0.1883 & 0.6639 & $0.028^{*}$ \\
\hline H11B & 0.6370 & 0.1205 & 0.7803 & $0.028 *$ \\
\hline $\mathrm{H} 11 \mathrm{C}$ & 0.6051 & 0.1850 & 0.9063 & $0.028 *$ \\
\hline N1 & $-0.08677(17)$ & $0.30611(7)$ & $0.7608(2)$ & $0.0154(3)$ \\
\hline $\mathrm{O} 1$ & $-0.08009(15)$ & $0.36827(7)$ & $0.7379(2)$ & 0.0252 \\
\hline $\mathrm{O} 2$ & $-0.20491(15)$ & $0.27751(7)$ & $0.7846(2)$ & 0.0236 \\
\hline $\mathrm{O} 3$ & $0.38271(14)$ & $0.38139(6)$ & $0.7701(2)$ & $0.0154(3)$ \\
\hline $\mathrm{O} 4$ & $0.33181(16)$ & $0.49408(7)$ & $0.7520(2)$ & $0.0257(3)$ \\
\hline $\mathrm{O} 5$ & $0.14161(14)$ & $0.08062(6)$ & $0.7543(2)$ & $0.0176(3)$ \\
\hline O6 & $0.41110(14)$ & $0.13243(6)$ & $0.7695(2)$ & $0.0164(3)$ \\
\hline
\end{tabular}


Atomic displacement parameters $\left(\AA^{2}\right)$

\begin{tabular}{lllllll}
\hline & $U^{11}$ & $U^{22}$ & $U^{33}$ & $U^{12}$ & $U^{13}$ & $U^{23}$ \\
\hline C1 & $0.0128(8)$ & $0.0155(8)$ & $0.0088(8)$ & $0.0001(6)$ & $0.0027(6)$ & $0.0011(6)$ \\
C2 & $0.0112(8)$ & $0.0174(8)$ & $0.0137(8)$ & $0.0022(6)$ & $0.0046(6)$ & $0.0009(6)$ \\
C3 & $0.0121(8)$ & $0.0174(8)$ & $0.0127(8)$ & $-0.0021(6)$ & $0.0037(6)$ & $0.0004(6)$ \\
C4 & $0.0147(8)$ & $0.0137(8)$ & $0.0133(8)$ & $-0.0031(6)$ & $0.0040(6)$ & $0.0003(6)$ \\
C5 & $0.0134(8)$ & $0.0152(8)$ & $0.0095(8)$ & $0.0014(6)$ & $0.0041(6)$ & $-0.0001(6)$ \\
C6 & $0.0113(8)$ & $0.0160(8)$ & $0.0117(8)$ & $-0.0008(6)$ & $0.0043(6)$ & $-0.0002(6)$ \\
C7 & $0.0105(8)$ & $0.0147(8)$ & $0.0177(9)$ & $-0.0003(6)$ & $0.0065(6)$ & $0.0002(6)$ \\
C8 & $0.0161(8)$ & $0.0149(8)$ & $0.0164(9)$ & $-0.0025(6)$ & $0.0063(7)$ & $-0.0006(6)$ \\
C9 & $0.0140(8)$ & $0.0182(9)$ & $0.0347(11)$ & $-0.0021(7)$ & $0.0094(8)$ & $-0.0009(8)$ \\
C10 & $0.0173(9)$ & $0.0163(8)$ & $0.0271(10)$ & $-0.0074(7)$ & $0.0075(7)$ & $-0.0016(7)$ \\
C11 & $0.0120(8)$ & $0.0170(8)$ & $0.0277(10)$ & $-0.0006(6)$ & $0.0065(7)$ & $-0.0011(7)$ \\
N1 & $0.0113(7)$ & $0.0171(7)$ & $0.0177(8)$ & $-0.0005(5)$ & $0.0041(6)$ & $-0.0007(5)$ \\
O1 & $0.0179(7)$ & $0.0159(6)$ & $0.0437(9)$ & $0.0031(5)$ & $0.0119(6)$ & $0.0037(6)$ \\
O2 & $0.0133(6)$ & $0.0236(7)$ & $0.0364(8)$ & $-0.0016(5)$ & $0.0111(6)$ & $0.0010(6)$ \\
O3 & $0.0114(6)$ & $0.0131(6)$ & $0.0227(7)$ & $-0.0011(4)$ & $0.0063(5)$ & $0.0000(5)$ \\
O4 & $0.0203(7)$ & $0.0141(6)$ & $0.0461(9)$ & $0.0008(5)$ & $0.0147(6)$ & $0.0018(6)$ \\
O5 & $0.0160(6)$ & $0.0120(6)$ & $0.0264(7)$ & $-0.0022(5)$ & $0.0085(5)$ & $-0.0001(5)$ \\
O6 & $0.0124(6)$ & $0.0137(6)$ & $0.0244(7)$ & $0.0013(5)$ & $0.0071(5)$ & $0.0005(5)$ \\
& & & & & &
\end{tabular}

Geometric parameters $\left(\AA,{ }^{\circ}\right)$

\begin{tabular}{llll}
\hline $\mathrm{C} 1-\mathrm{C} 2$ & $1.399(2)$ & $\mathrm{C} 8-\mathrm{O} 3$ & $1.356(2)$ \\
$\mathrm{C} 1-\mathrm{C} 6$ & $1.405(2)$ & $\mathrm{C} 8-\mathrm{C} 9$ & $1.494(2)$ \\
$\mathrm{C} 1-\mathrm{C} 7$ & $1.512(2)$ & $\mathrm{C} 9-\mathrm{H} 9 \mathrm{~A}$ & 0.9800 \\
$\mathrm{C} 2-\mathrm{C} 3$ & $1.401(2)$ & $\mathrm{C} 9-\mathrm{H} 9 \mathrm{~B}$ & 0.9800 \\
$\mathrm{C} 2-\mathrm{N} 1$ & $1.456(2)$ & $\mathrm{C} 9-\mathrm{H} 9 \mathrm{C}$ & 0.9800 \\
$\mathrm{C} 3-\mathrm{C} 4$ & $1.377(2)$ & $\mathrm{C} 10-\mathrm{O} 5$ & $0.436(2)$ \\
$\mathrm{C} 3-\mathrm{H} 3$ & 0.9500 & $\mathrm{C} 10-\mathrm{H} 10 \mathrm{~A}$ & 0.9800 \\
$\mathrm{C} 4-\mathrm{O} 5$ & $1.361(2)$ & $\mathrm{C} 10-\mathrm{H} 10 \mathrm{~B}$ & 0.9800 \\
$\mathrm{C} 4-\mathrm{C} 5$ & $1.420(2)$ & $\mathrm{C} 10-\mathrm{H} 10 \mathrm{C}$ & $1.446(2)$ \\
$\mathrm{C} 5-\mathrm{O} 6$ & $1.351(2)$ & $\mathrm{C} 11-\mathrm{O} 6$ & 0.9800 \\
$\mathrm{C} 5-\mathrm{C} 6$ & $1.388(2)$ & $\mathrm{C} 11-\mathrm{H} 11 \mathrm{~A}$ & 0.9800 \\
$\mathrm{C} 6-\mathrm{H} 6$ & 0.9500 & $\mathrm{C} 11-\mathrm{H} 11 \mathrm{~B}$ & 0.9800 \\
$\mathrm{C} 7-\mathrm{O} 3$ & $1.4419(19)$ & $\mathrm{C} 11-\mathrm{H} 11 \mathrm{C}$ & $1.229(2)$ \\
$\mathrm{C} 7-\mathrm{H} 7 \mathrm{~A}$ & 0.9900 & $\mathrm{~N} 1-\mathrm{O} 1$ & $1.2390(19)$ \\
$\mathrm{C} 7-\mathrm{H} 7 \mathrm{~B}$ & 0.9900 & $\mathrm{~N} 1-\mathrm{O} 2$ & $110.96(15)$ \\
$\mathrm{C} 8-\mathrm{O} 4$ & $1.204(2)$ & & 109.5 \\
& & & 109.5 \\
$\mathrm{C} 2-\mathrm{C} 1-\mathrm{C} 6$ & $116.20(15)$ & $\mathrm{O} 3-\mathrm{C} 8-\mathrm{C} 9$ & 109.5 \\
$\mathrm{C} 2-\mathrm{C} 1-\mathrm{C} 7$ & $124.21(15)$ & $\mathrm{C} 8-\mathrm{C} 9-\mathrm{H} 9 \mathrm{~A}$ & 109.5 \\
$\mathrm{C} 6-\mathrm{C} 1-\mathrm{C} 7$ & $119.59(15)$ & $\mathrm{C} 8-\mathrm{C} 9-\mathrm{H} 9 \mathrm{~B}$ & 109.5 \\
$\mathrm{C} 1-\mathrm{C} 2-\mathrm{C} 3$ & $122.90(15)$ & $\mathrm{H} 9 \mathrm{C}-\mathrm{C} 9-\mathrm{H} 9 \mathrm{~B}$ & 109.5 \\
$\mathrm{C} 1-\mathrm{C} 2-\mathrm{N} 1$ & $121.08(15)$ & $\mathrm{C} 8-\mathrm{C} 9-\mathrm{H} 9 \mathrm{C}$ & \\
$\mathrm{C} 3-\mathrm{C} 2-\mathrm{N} 1$ & $116.02(15)$ & $\mathrm{H} 9 \mathrm{~A}-\mathrm{C} 9-\mathrm{H} 9 \mathrm{C}$ & \\
$\mathrm{C} 4-\mathrm{C} 3-\mathrm{C} 2$ & $119.83(15)$ & $\mathrm{H} 9 \mathrm{~B}-\mathrm{C} 9-\mathrm{H} 9 \mathrm{C}$ &
\end{tabular}




\begin{tabular}{|c|c|c|c|}
\hline $\mathrm{C} 4-\mathrm{C} 3-\mathrm{H} 3$ & 120.1 & $\mathrm{O} 5-\mathrm{C} 10-\mathrm{H} 10 \mathrm{~A}$ & 109.5 \\
\hline $\mathrm{C} 2-\mathrm{C} 3-\mathrm{H} 3$ & 120.1 & $\mathrm{O} 5-\mathrm{C} 10-\mathrm{H} 10 \mathrm{~B}$ & 109.5 \\
\hline $\mathrm{O} 5-\mathrm{C} 4-\mathrm{C} 3$ & $125.67(15)$ & $\mathrm{H} 10 \mathrm{~A}-\mathrm{C} 10-\mathrm{H} 10 \mathrm{~B}$ & 109.5 \\
\hline $\mathrm{O} 5-\mathrm{C} 4-\mathrm{C} 5$ & $115.43(15)$ & $\mathrm{O} 5-\mathrm{C} 10-\mathrm{H} 10 \mathrm{C}$ & 109.5 \\
\hline $\mathrm{C} 3-\mathrm{C} 4-\mathrm{C} 5$ & $118.90(15)$ & $\mathrm{H} 10 \mathrm{~A}-\mathrm{C} 10-\mathrm{H} 10 \mathrm{C}$ & 109.5 \\
\hline $\mathrm{O} 6-\mathrm{C} 5-\mathrm{C} 6$ & $125.00(15)$ & $\mathrm{H} 10 \mathrm{~B}-\mathrm{C} 10-\mathrm{H} 10 \mathrm{C}$ & 109.5 \\
\hline $\mathrm{O} 6-\mathrm{C} 5-\mathrm{C} 4$ & $114.87(15)$ & $\mathrm{O} 6-\mathrm{C} 11-\mathrm{H} 11 \mathrm{~A}$ & 109.5 \\
\hline $\mathrm{C} 6-\mathrm{C} 5-\mathrm{C} 4$ & $120.12(15)$ & $\mathrm{O} 6-\mathrm{C} 11-\mathrm{H} 11 \mathrm{~B}$ & 109.5 \\
\hline $\mathrm{C} 5-\mathrm{C} 6-\mathrm{C} 1$ & $122.03(15)$ & $\mathrm{H} 11 \mathrm{~A}-\mathrm{C} 11-\mathrm{H} 11 \mathrm{~B}$ & 109.5 \\
\hline $\mathrm{C} 5-\mathrm{C} 6-\mathrm{H} 6$ & 119.0 & $\mathrm{O} 6-\mathrm{C} 11-\mathrm{H} 11 \mathrm{C}$ & 109.5 \\
\hline $\mathrm{C} 1-\mathrm{C} 6-\mathrm{H} 6$ & 119.0 & $\mathrm{H} 11 \mathrm{~A}-\mathrm{C} 11-\mathrm{H} 11 \mathrm{C}$ & 109.5 \\
\hline $\mathrm{O} 3-\mathrm{C} 7-\mathrm{C} 1$ & $107.82(13)$ & $\mathrm{H} 11 \mathrm{~B}-\mathrm{C} 11-\mathrm{H} 11 \mathrm{C}$ & 109.5 \\
\hline $\mathrm{O} 3-\mathrm{C} 7-\mathrm{H} 7 \mathrm{~A}$ & 110.1 & $\mathrm{O} 1-\mathrm{N} 1-\mathrm{O} 2$ & $122.43(15)$ \\
\hline $\mathrm{C} 1-\mathrm{C} 7-\mathrm{H} 7 \mathrm{~A}$ & 110.1 & $\mathrm{O} 1-\mathrm{N} 1-\mathrm{C} 2$ & $119.04(14)$ \\
\hline $\mathrm{O} 3-\mathrm{C} 7-\mathrm{H} 7 \mathrm{~B}$ & 110.1 & $\mathrm{O} 2-\mathrm{N} 1-\mathrm{C} 2$ & $118.53(14)$ \\
\hline $\mathrm{C} 1-\mathrm{C} 7-\mathrm{H} 7 \mathrm{~B}$ & 110.1 & $\mathrm{C} 8-\mathrm{O} 3-\mathrm{C} 7$ & $114.47(13)$ \\
\hline $\mathrm{H} 7 \mathrm{~A}-\mathrm{C} 7-\mathrm{H} 7 \mathrm{~B}$ & 108.5 & $\mathrm{C} 4-\mathrm{O} 5-\mathrm{C} 10$ & $116.93(13)$ \\
\hline $\mathrm{O} 4-\mathrm{C} 8-\mathrm{O} 3$ & $122.58(16)$ & $\mathrm{C} 5-\mathrm{O} 6-\mathrm{C} 11$ & $117.20(13)$ \\
\hline $\mathrm{O} 4-\mathrm{C} 8-\mathrm{C} 9$ & $126.45(16)$ & & \\
\hline $\mathrm{C} 6-\mathrm{C} 1-\mathrm{C} 2-\mathrm{C} 3$ & $-0.7(2)$ & $\mathrm{C} 7-\mathrm{C} 1-\mathrm{C} 6-\mathrm{C} 5$ & $-179.55(15)$ \\
\hline $\mathrm{C} 7-\mathrm{C} 1-\mathrm{C} 2-\mathrm{C} 3$ & $179.08(16)$ & $\mathrm{C} 2-\mathrm{C} 1-\mathrm{C} 7-\mathrm{O} 3$ & $-179.18(15)$ \\
\hline $\mathrm{C} 6-\mathrm{C} 1-\mathrm{C} 2-\mathrm{N} 1$ & $178.95(15)$ & $\mathrm{C} 6-\mathrm{C} 1-\mathrm{C} 7-\mathrm{O} 3$ & $0.6(2)$ \\
\hline $\mathrm{C} 7-\mathrm{C} 1-\mathrm{C} 2-\mathrm{N} 1$ & $-1.2(3)$ & $\mathrm{C} 1-\mathrm{C} 2-\mathrm{N} 1-\mathrm{O} 1$ & $9.5(2)$ \\
\hline $\mathrm{C} 1-\mathrm{C} 2-\mathrm{C} 3-\mathrm{C} 4$ & $0.1(3)$ & $\mathrm{C} 3-\mathrm{C} 2-\mathrm{N} 1-\mathrm{O} 1$ & $-170.83(16)$ \\
\hline $\mathrm{N} 1-\mathrm{C} 2-\mathrm{C} 3-\mathrm{C} 4$ & $-179.59(15)$ & $\mathrm{C} 1-\mathrm{C} 2-\mathrm{N} 1-\mathrm{O} 2$ & $-170.13(16)$ \\
\hline $\mathrm{C} 2-\mathrm{C} 3-\mathrm{C} 4-\mathrm{O} 5$ & $-179.39(16)$ & $\mathrm{C} 3-\mathrm{C} 2-\mathrm{N} 1-\mathrm{O} 2$ & $9.6(2)$ \\
\hline $\mathrm{C} 2-\mathrm{C} 3-\mathrm{C} 4-\mathrm{C} 5$ & $1.0(2)$ & $\mathrm{O} 4-\mathrm{C} 8-\mathrm{O} 3-\mathrm{C} 7$ & $2.5(2)$ \\
\hline $\mathrm{O} 5-\mathrm{C} 4-\mathrm{C} 5-\mathrm{O} 6$ & $0.1(2)$ & $\mathrm{C} 9-\mathrm{C} 8-\mathrm{O} 3-\mathrm{C} 7$ & $-176.69(15)$ \\
\hline $\mathrm{C} 3-\mathrm{C} 4-\mathrm{C} 5-\mathrm{O} 6$ & $179.78(15)$ & $\mathrm{C} 1-\mathrm{C} 7-\mathrm{O} 3-\mathrm{C} 8$ & $175.79(14)$ \\
\hline $\mathrm{O} 5-\mathrm{C} 4-\mathrm{C} 5-\mathrm{C} 6$ & $178.91(14)$ & $\mathrm{C} 3-\mathrm{C} 4-\mathrm{O} 5-\mathrm{C} 10$ & $2.4(3)$ \\
\hline $\mathrm{C} 3-\mathrm{C} 4-\mathrm{C} 5-\mathrm{C} 6$ & $-1.4(2)$ & $\mathrm{C} 5-\mathrm{C} 4-\mathrm{O} 5-\mathrm{C} 10$ & $-177.96(15)$ \\
\hline $\mathrm{O} 6-\mathrm{C} 5-\mathrm{C} 6-\mathrm{C} 1$ & $179.47(15)$ & $\mathrm{C} 6-\mathrm{C} 5-\mathrm{O} 6-\mathrm{C} 11$ & $2.1(2)$ \\
\hline $\mathrm{C} 4-\mathrm{C} 5-\mathrm{C} 6-\mathrm{C} 1$ & $0.8(2)$ & $\mathrm{C} 4-\mathrm{C} 5-\mathrm{O} 6-\mathrm{C} 11$ & $-179.17(15)$ \\
\hline $\mathrm{C} 2-\mathrm{C} 1-\mathrm{C} 6-\mathrm{C} 5$ & $0.3(2)$ & & \\
\hline
\end{tabular}

Hydrogen-bond geometry $\left(A,{ }^{\circ}\right)$

\begin{tabular}{lllll}
\hline$D-\mathrm{H} \cdots A$ & $D-\mathrm{H}$ & $\mathrm{H} \cdots A$ & $D \cdots A$ & $D-\mathrm{H} \cdots A$ \\
\hline $\mathrm{C} 11-\mathrm{H} 11 B \cdots \mathrm{O} 4^{\mathrm{i}}$ & 0.98 & 2.50 & $3.369(2)$ & 147 \\
\hline
\end{tabular}

Symmetry code: (i) $-x+1, y-1 / 2,-z+3 / 2$.

(yellow) 4,5-Dimethoxy-2-nitrobenzyl Acetate

Crystal data

$\mathrm{C}_{11} \mathrm{H}_{13} \mathrm{NO}_{6}$

$M_{r}=255.22$

Hall symbol: -P 2ybc

Monoclinic, $P 2_{1} / c$

$a=10.476(3) \AA$

$b=10.714(3) \AA$ 
$c=10.266(3) \AA$

$\beta=105.077(10)^{\circ}$

$V=1112.6(6) \AA^{3}$

$Z=4$

$F(000)=536$

$D_{\mathrm{x}}=1.52 \mathrm{Mg} \mathrm{m}^{-3}$

Mo $K \alpha$ radiation, $\lambda=0.71069 \AA$

Data collection

Rigaku Mercury375R (2x2 bin mode) diffractometer

Radiation source: fine-focus sealed tube Graphite monochromator

Detector resolution: 13.6612 pixels $\mathrm{mm}^{-1}$

profile data from $\omega$-scan

Absorption correction: multi-scan (REQAB; Rigaku, 1998)

$T_{\min }=0.797, T_{\max }=0.970$

\section{Refinement}

Refinement on $F^{2}$

Least-squares matrix: full

$R\left[F^{2}>2 \sigma\left(F^{2}\right)\right]=0.049$

$w R\left(F^{2}\right)=0.130$

$S=1.13$

2058 reflections

166 parameters

0 restraints

Primary atom site location: structure-invariant direct methods
Cell parameters from 2424 reflections

$\theta=3.1-27.5^{\circ}$

$\mu=0.13 \mathrm{~mm}^{-1}$

$T=93 \mathrm{~K}$

Neecle, yellow

$0.56 \times 0.54 \times 0.25 \mathrm{~mm}$

9498 measured reflections

2058 independent reflections

1769 reflections with $I>2 \sigma(I)$

$R_{\text {int }}=0.033$

$\theta_{\text {max }}=25.5^{\circ}, \theta_{\min }=3.1^{\circ}$

$h=-12 \rightarrow 12$

$k=-12 \rightarrow 12$

$l=-12 \rightarrow 12$

Secondary atom site location: difference Fourier map

Hydrogen site location: inferred from neighbouring sites

$\mathrm{H}$-atom parameters not refined

$w=1 /\left[\sigma^{2}\left(F_{\mathrm{o}}^{2}\right)+(0.0689 P)^{2}+0.4454 P\right]$

where $P=\left(F_{\mathrm{o}}{ }^{2}+2 F_{\mathrm{c}}{ }^{2}\right) / 3$

$(\Delta / \sigma)_{\max }<0.001$

$\Delta \rho_{\max }=0.38$ e $\AA^{-3}$

$\Delta \rho_{\min }=-0.35$ e $\AA^{-3}$

\section{Special details}

Experimental. Rigaku (1998). REQAB. Rigaku Corporation, Tokyo, Japan.

Geometry. All e.s.d.'s (except the e.s.d. in the dihedral angle between two 1.s. planes) are estimated using the full covariance matrix. The cell e.s.d.'s are taken into account individually in the estimation of e.s.d.'s in distances, angles and torsion angles; correlations between e.s.d.'s in cell parameters are only used when they are defined by crystal symmetry. An approximate (isotropic) treatment of cell e.s.d.'s is used for estimating e.s.d.'s involving 1.s. planes.

Refinement. Refinement of $F^{2}$ against ALL reflections. The weighted $R$-factor $w R$ and goodness of fit $S$ are based on $F^{2}$, conventional $R$-factors $R$ are based on $F$, with $F$ set to zero for negative $F^{2}$. The threshold expression of $F^{2}>\sigma\left(F^{2}\right)$ is used only for calculating $R$-factors(gt) etc. and is not relevant to the choice of reflections for refinement. $R$-factors based on $F^{2}$ are statistically about twice as large as those based on $F$, and $R$ - factors based on ALL data will be even larger.

Fractional atomic coordinates and isotropic or equivalent isotropic displacement parameters $\left(\AA^{2}\right)$

\begin{tabular}{lllll}
\hline & $x$ & $y$ & $z$ & $U_{\text {iso }} * / U_{\text {eq }}$ \\
\hline C1 & $0.22297(15)$ & $0.82305(16)$ & $0.58154(16)$ & $0.0158(4)$ \\
C2 & $0.29963(16)$ & $0.88543(15)$ & $0.69423(17)$ & $0.0154(4)$ \\
C3 & $0.37895(16)$ & $0.82230(16)$ & $0.80564(16)$ & $0.0168(4)$ \\
H3 & 0.4307 & 0.8681 & 0.8801 & $0.020^{*}$ \\
C4 & $0.38181(16)$ & $0.69396(16)$ & $0.80717(16)$ & $0.0167(4)$ \\
C5 & $0.30317(15)$ & $0.62775(16)$ & $0.69555(17)$ & $0.0154(4)$ \\
C6 & $0.22676(16)$ & $0.69258(16)$ & $0.58567(16)$ & $0.0158(4)$ \\
H6 & 0.1754 & 0.6469 & 0.5109 & $0.019 *$
\end{tabular}




$\begin{array}{lllll}\text { C7 } & 0.13844(16) & 0.88840(15) & 0.45857(17) & 0.0166(4) \\ \text { H7A } & 0.0754 & 0.9456 & 0.4852 & 0.020^{*} \\ \text { H7B } & 0.1948 & 0.9379 & 0.4140 & 0.020^{*} \\ \text { C8 } & -0.01205(16) & 0.83679(16) & 0.25084(16) & 0.0175(4) \\ \text { C9 } & -0.08377(18) & 0.73342(16) & 0.16403(18) & 0.0216(4) \\ \text { H9A } & -0.1781 & 0.7378 & 0.1603 & 0.032^{*} \\ \text { H9B } & -0.0481 & 0.6529 & 0.2022 & 0.032^{*} \\ \text { H9C } & -0.0721 & 0.7418 & 0.0728 & 0.032^{*} \\ \text { C10 } & 0.53711(16) & 0.68572(16) & 1.02246(16) & 0.0186(4) \\ \text { H10A } & 0.6026 & 0.7354 & 0.9923 & 0.028^{*} \\ \text { H10B } & 0.5826 & 0.6245 & 1.0895 & 0.028^{*} \\ \text { H10C } & 0.4834 & 0.7409 & 1.0630 & 0.028^{*} \\ \text { C11 } & 0.22990(18) & 0.43083(16) & 0.59702(17) & 0.0211(4) \\ \text { H11A } & 0.1368 & 0.4538 & 0.5832 & 0.032^{*} \\ \text { H11B } & 0.2410 & 0.3417 & 0.6185 & 0.032^{*} \\ \text { H11C } & 0.2574 & 0.4480 & 0.5146 & 0.032^{*} \\ \text { O1 } & 0.23683(12) & 1.08224(11) & 0.60592(12) & 0.0219(3) \\ \text { O2 } & 0.36433(12) & 1.07109(11) & 0.80903(12) & 0.0228(3) \\ \text { O3 } & 0.06713(11) & 0.79354(11) & 0.36698(12) & 0.0186(3) \\ \text { O4 } & -0.02320(12) & 0.94638(11) & 0.22241(12) & 0.0229(3) \\ \text { O5 } & 0.45330(11) & 0.62196(11) & 0.90924(12) & 0.0183(3) \\ \text { O6 } & 0.30975(12) & 0.50251(11) & 0.70663(12) & 0.0187(3) \\ \text { N1 } & 0.30069(14) & 1.02142(14) & 0.70366(14) & 0.0175(3) \\ & & & & \end{array}$

Atomic displacement parameters $\left(\AA^{2}\right)$

\begin{tabular}{lllllll}
\hline & $U^{11}$ & $U^{22}$ & $U^{33}$ & $U^{12}$ & $U^{13}$ & $U^{23}$ \\
\hline C1 & $0.0163(8)$ & $0.0161(8)$ & $0.0155(8)$ & $0.0025(6)$ & $0.0052(7)$ & $0.0004(6)$ \\
C2 & $0.0196(8)$ & $0.0084(8)$ & $0.0189(9)$ & $0.0000(6)$ & $0.0064(7)$ & $-0.0011(6)$ \\
C3 & $0.0179(8)$ & $0.0159(8)$ & $0.0154(8)$ & $-0.0026(7)$ & $0.0023(7)$ & $-0.0015(6)$ \\
C4 & $0.0189(8)$ & $0.0155(9)$ & $0.0149(8)$ & $0.0008(7)$ & $0.0031(7)$ & $0.0008(6)$ \\
C5 & $0.0163(8)$ & $0.0136(9)$ & $0.0158(8)$ & $-0.0006(6)$ & $0.0033(7)$ & $-0.0002(6)$ \\
C6 & $0.0177(8)$ & $0.0137(9)$ & $0.0153(8)$ & $-0.0009(6)$ & $0.0033(7)$ & $-0.0023(6)$ \\
C7 & $0.0193(8)$ & $0.0115(8)$ & $0.0163(8)$ & $-0.0006(6)$ & $0.0000(7)$ & $-0.0022(6)$ \\
C8 & $0.0169(8)$ & $0.0188(9)$ & $0.0148(8)$ & $0.0001(7)$ & $0.0006(7)$ & $0.0014(7)$ \\
C9 & $0.0233(9)$ & $0.0158(9)$ & $0.0212(9)$ & $0.0007(7)$ & $-0.0025(7)$ & $0.0003(7)$ \\
C10 & $0.0199(8)$ & $0.0181(9)$ & $0.0144(8)$ & $-0.0017(7)$ & $-0.0018(7)$ & $-0.0009(7)$ \\
C11 & $0.0278(9)$ & $0.0134(9)$ & $0.0188(9)$ & $-0.0016(7)$ & $0.0000(7)$ & $-0.0029(6)$ \\
O1 & $0.0277(7)$ & $0.0145(6)$ & $0.0204(7)$ & $0.0032(5)$ & $0.0009(5)$ & $0.0035(5)$ \\
O2 & $0.0307(7)$ & $0.0153(7)$ & $0.0189(7)$ & $-0.0016(5)$ & $-0.0001(5)$ & $-0.0052(5)$ \\
O3 & $0.0215(6)$ & $0.0127(6)$ & $0.0173(6)$ & $0.0004(5)$ & $-0.0027(5)$ & $-0.0002(5)$ \\
O4 & $0.0277(7)$ & $0.0140(6)$ & $0.0229(7)$ & $0.0001(5)$ & $-0.0008(5)$ & $0.0031(5)$ \\
O5 & $0.0222(6)$ & $0.0131(6)$ & $0.0146(6)$ & $-0.0007(5)$ & $-0.0040(5)$ & $0.0008(5)$ \\
O6 & $0.0245(6)$ & $0.0095(6)$ & $0.0183(6)$ & $-0.0001(5)$ & $-0.0013(5)$ & $-0.0001(4)$ \\
N1 & $0.0195(7)$ & $0.0153(8)$ & $0.0168(7)$ & $-0.0007(6)$ & $0.0029(6)$ & $-0.0007(6)$ \\
& & & & & & \\
\hline
\end{tabular}


Geometric parameters $\left(\AA,{ }^{\circ}\right)$

\begin{tabular}{|c|c|c|c|}
\hline $\mathrm{C} 1-\mathrm{C} 2$ & $1.395(2)$ & $\mathrm{C} 8-\mathrm{O} 3$ & $1.345(2)$ \\
\hline $\mathrm{C} 1-\mathrm{C} 6$ & $1.399(2)$ & $\mathrm{C} 8-\mathrm{C} 9$ & $1.496(2)$ \\
\hline $\mathrm{C} 1-\mathrm{C} 7$ & $1.512(2)$ & C9-H9A & 0.9800 \\
\hline $\mathrm{C} 2-\mathrm{C} 3$ & $1.401(2)$ & C9-H9B & 0.9800 \\
\hline $\mathrm{C} 2-\mathrm{N} 1$ & $1.460(2)$ & $\mathrm{C} 9-\mathrm{H} 9 \mathrm{C}$ & 0.9800 \\
\hline $\mathrm{C} 3-\mathrm{C} 4$ & $1.375(3)$ & $\mathrm{C} 10-\mathrm{O} 5$ & $1.4344(19)$ \\
\hline $\mathrm{C} 3-\mathrm{H} 3$ & 0.9500 & $\mathrm{C} 10-\mathrm{H} 10 \mathrm{~A}$ & 0.9800 \\
\hline $\mathrm{C} 4-\mathrm{O} 5$ & $1.359(2)$ & $\mathrm{C} 10-\mathrm{H} 10 \mathrm{~B}$ & 0.9800 \\
\hline $\mathrm{C} 4-\mathrm{C} 5$ & $1.416(2)$ & $\mathrm{C} 10-\mathrm{H} 10 \mathrm{C}$ & 0.9800 \\
\hline $\mathrm{C} 5-\mathrm{O} 6$ & $1.347(2)$ & $\mathrm{C} 11-\mathrm{O} 6$ & $1.437(2)$ \\
\hline $\mathrm{C} 5-\mathrm{C} 6$ & $1.388(2)$ & $\mathrm{C} 11-\mathrm{H} 11 \mathrm{~A}$ & 0.9800 \\
\hline $\mathrm{C} 6-\mathrm{H} 6$ & 0.9500 & $\mathrm{C} 11-\mathrm{H} 11 \mathrm{~B}$ & 0.9800 \\
\hline $\mathrm{C} 7-\mathrm{O} 3$ & $1.4524(19)$ & $\mathrm{C} 11-\mathrm{H} 11 \mathrm{C}$ & 0.9800 \\
\hline $\mathrm{C} 7-\mathrm{H} 7 \mathrm{~A}$ & 0.9900 & $\mathrm{O} 1-\mathrm{N} 1$ & $1.2368(19)$ \\
\hline C7-H7B & 0.9900 & $\mathrm{O} 2-\mathrm{N} 1$ & $1.2339(19)$ \\
\hline $\mathrm{C} 8-\mathrm{O} 4$ & $1.208(2)$ & & \\
\hline $\mathrm{C} 2-\mathrm{C} 1-\mathrm{C} 6$ & $116.61(15)$ & $\mathrm{O} 3-\mathrm{C} 8-\mathrm{C} 9$ & $111.81(14)$ \\
\hline $\mathrm{C} 2-\mathrm{C} 1-\mathrm{C} 7$ & $123.79(16)$ & $\mathrm{C} 8-\mathrm{C} 9-\mathrm{H} 9 \mathrm{~A}$ & 109.5 \\
\hline $\mathrm{C} 6-\mathrm{C} 1-\mathrm{C} 7$ & $119.60(14)$ & $\mathrm{C} 8-\mathrm{C} 9-\mathrm{H} 9 \mathrm{~B}$ & 109.5 \\
\hline $\mathrm{C} 1-\mathrm{C} 2-\mathrm{C} 3$ & $122.48(16)$ & $\mathrm{H} 9 \mathrm{~A}-\mathrm{C} 9-\mathrm{H} 9 \mathrm{~B}$ & 109.5 \\
\hline $\mathrm{C} 1-\mathrm{C} 2-\mathrm{N} 1$ & $121.72(15)$ & $\mathrm{C} 8-\mathrm{C} 9-\mathrm{H} 9 \mathrm{C}$ & 109.5 \\
\hline $\mathrm{C} 3-\mathrm{C} 2-\mathrm{N} 1$ & $115.79(15)$ & $\mathrm{H} 9 \mathrm{~A}-\mathrm{C} 9-\mathrm{H} 9 \mathrm{C}$ & 109.5 \\
\hline $\mathrm{C} 4-\mathrm{C} 3-\mathrm{C} 2$ & $119.91(15)$ & $\mathrm{H} 9 \mathrm{~B}-\mathrm{C} 9-\mathrm{H} 9 \mathrm{C}$ & 109.5 \\
\hline $\mathrm{C} 4-\mathrm{C} 3-\mathrm{H} 3$ & 120.0 & $\mathrm{O} 5-\mathrm{C} 10-\mathrm{H} 10 \mathrm{~A}$ & 109.5 \\
\hline $\mathrm{C} 2-\mathrm{C} 3-\mathrm{H} 3$ & 120.0 & $\mathrm{O} 5-\mathrm{C} 10-\mathrm{H} 10 \mathrm{~B}$ & 109.5 \\
\hline $\mathrm{O} 5-\mathrm{C} 4-\mathrm{C} 3$ & $125.62(15)$ & $\mathrm{H} 10 \mathrm{~A}-\mathrm{C} 10-\mathrm{H} 10 \mathrm{~B}$ & 109.5 \\
\hline $\mathrm{O} 5-\mathrm{C} 4-\mathrm{C} 5$ & $115.33(15)$ & $\mathrm{O} 5-\mathrm{C} 10-\mathrm{H} 10 \mathrm{C}$ & 109.5 \\
\hline $\mathrm{C} 3-\mathrm{C} 4-\mathrm{C} 5$ & $119.04(15)$ & $\mathrm{H} 10 \mathrm{~A}-\mathrm{C} 10-\mathrm{H} 10 \mathrm{C}$ & 109.5 \\
\hline $\mathrm{O} 6-\mathrm{C} 5-\mathrm{C} 6$ & $124.98(15)$ & $\mathrm{H} 10 \mathrm{~B}-\mathrm{C} 10-\mathrm{H} 10 \mathrm{C}$ & 109.5 \\
\hline $\mathrm{O} 6-\mathrm{C} 5-\mathrm{C} 4$ & $115.13(14)$ & $\mathrm{O} 6-\mathrm{C} 11-\mathrm{H} 11 \mathrm{~A}$ & 109.5 \\
\hline $\mathrm{C} 6-\mathrm{C} 5-\mathrm{C} 4$ & $119.89(16)$ & $\mathrm{O} 6-\mathrm{C} 11-\mathrm{H} 11 \mathrm{~B}$ & 109.5 \\
\hline $\mathrm{C} 5-\mathrm{C} 6-\mathrm{C} 1$ & $122.05(15)$ & $\mathrm{H} 11 \mathrm{~A}-\mathrm{C} 11-\mathrm{H} 11 \mathrm{~B}$ & 109.5 \\
\hline $\mathrm{C} 5-\mathrm{C} 6-\mathrm{H} 6$ & 119.0 & $\mathrm{O} 6-\mathrm{C} 11-\mathrm{H} 11 \mathrm{C}$ & 109.5 \\
\hline $\mathrm{C} 1-\mathrm{C} 6-\mathrm{H} 6$ & 119.0 & $\mathrm{H} 11 \mathrm{~A}-\mathrm{C} 11-\mathrm{H} 11 \mathrm{C}$ & 109.5 \\
\hline $\mathrm{O} 3-\mathrm{C} 7-\mathrm{C} 1$ & $107.90(13)$ & $\mathrm{H} 11 \mathrm{~B}-\mathrm{C} 11-\mathrm{H} 11 \mathrm{C}$ & 109.5 \\
\hline $\mathrm{O} 3-\mathrm{C} 7-\mathrm{H} 7 \mathrm{~A}$ & 110.1 & $\mathrm{C} 8-\mathrm{O} 3-\mathrm{C} 7$ & $115.31(13)$ \\
\hline $\mathrm{C} 1-\mathrm{C} 7-\mathrm{H} 7 \mathrm{~A}$ & 110.1 & $\mathrm{C} 4-\mathrm{O} 5-\mathrm{C} 10$ & $116.94(13)$ \\
\hline $\mathrm{O} 3-\mathrm{C} 7-\mathrm{H} 7 \mathrm{~B}$ & 110.1 & $\mathrm{C} 5-\mathrm{O} 6-\mathrm{C} 11$ & $117.37(13)$ \\
\hline $\mathrm{C} 1-\mathrm{C} 7-\mathrm{H} 7 \mathrm{~B}$ & 110.1 & $\mathrm{O} 2-\mathrm{N} 1-\mathrm{O} 1$ & $122.61(15)$ \\
\hline $\mathrm{H} 7 \mathrm{~A}-\mathrm{C} 7-\mathrm{H} 7 \mathrm{~B}$ & 108.4 & $\mathrm{O} 2-\mathrm{N} 1-\mathrm{C} 2$ & $118.78(14)$ \\
\hline $\mathrm{O} 4-\mathrm{C} 8-\mathrm{O} 3$ & $123.19(15)$ & $\mathrm{O} 1-\mathrm{N} 1-\mathrm{C} 2$ & $118.60(13)$ \\
\hline $\mathrm{O} 4-\mathrm{C} 8-\mathrm{C} 9$ & $125.01(15)$ & & \\
\hline $\mathrm{C} 6-\mathrm{C} 1-\mathrm{C} 2-\mathrm{C} 3$ & $1.2(2)$ & $\mathrm{C} 7-\mathrm{C} 1-\mathrm{C} 6-\mathrm{C} 5$ & $179.61(14)$ \\
\hline $\mathrm{C} 7-\mathrm{C} 1-\mathrm{C} 2-\mathrm{C} 3$ & $-178.75(15)$ & $\mathrm{C} 2-\mathrm{C} 1-\mathrm{C} 7-\mathrm{O} 3$ & $-176.32(14)$ \\
\hline
\end{tabular}




$\begin{array}{llll}\mathrm{C} 6-\mathrm{C} 1-\mathrm{C} 2-\mathrm{N} 1 & -178.07(14) & \mathrm{C} 6-\mathrm{C} 1-\mathrm{C} 7-\mathrm{O} 3 & 3.7(2) \\ \mathrm{C} 7-\mathrm{C} 1-\mathrm{C} 2-\mathrm{N} 1 & 2.0(2) & \mathrm{O} 4-\mathrm{C} 8-\mathrm{O} 3-\mathrm{C} 7 & 0.8(2) \\ \mathrm{C} 1-\mathrm{C} 2-\mathrm{C} 3-\mathrm{C} 4 & -0.8(2) & \mathrm{C} 9-\mathrm{C} 8-\mathrm{O} 3-\mathrm{C} 7 & -178.75(13) \\ \mathrm{N} 1-\mathrm{C} 2-\mathrm{C} 3-\mathrm{C} 4 & 178.48(15) & \mathrm{C} 1-\mathrm{C} 7-\mathrm{O} 3-\mathrm{C} 8 & -2.8(2) \\ \mathrm{C} 2-\mathrm{C} 3-\mathrm{C} 4-\mathrm{O} 5 & -179.33(14) & \mathrm{C} 3-\mathrm{C} 4-\mathrm{O} 5-\mathrm{C} 10 & 178.28(13) \\ \mathrm{C} 2-\mathrm{C} 3-\mathrm{C} 4-\mathrm{C} 5 & -0.4(2) & \mathrm{C} 5-\mathrm{C} 4-\mathrm{O} 5-\mathrm{C} 10 & -1.0(2) \\ \mathrm{O} 5-\mathrm{C} 4-\mathrm{C} 5-\mathrm{O} 6 & 0.4(2) & \mathrm{C} 6-\mathrm{C} 5-\mathrm{O} 6-\mathrm{C} 11 & 178.91(13) \\ \mathrm{C} 3-\mathrm{C} 4-\mathrm{C} 5-\mathrm{O} 6 & -178.65(15) & \mathrm{C} 4-\mathrm{C} 5-\mathrm{O} 6-\mathrm{C} 11 & 175.79(14) \\ \mathrm{O} 5-\mathrm{C} 4-\mathrm{C} 5-\mathrm{C} 6 & -179.73(14) & \mathrm{C} 1-\mathrm{C} 2-\mathrm{N} 1-\mathrm{O} 2 & -3.5(2) \\ \mathrm{C} 3-\mathrm{C} 4-\mathrm{C} 5-\mathrm{C} 6 & 1.3(2) & \mathrm{C} 3-\mathrm{C} 2-\mathrm{N} 1-\mathrm{O} 2 & -3.7(2) \\ \mathrm{O} 6-\mathrm{C} 5-\mathrm{C} 6-\mathrm{C} 1 & 179.03(15) & \mathrm{C} 1-\mathrm{C} 2-\mathrm{N} 1-\mathrm{O} 1 & 177.02(14) \\ \mathrm{C} 4-\mathrm{C} 5-\mathrm{C} 6-\mathrm{C} 1 & -0.9(2) & \mathrm{C} 3-\mathrm{C} 2-\mathrm{N} 1-\mathrm{O} 1 & \\ \mathrm{C} 2-\mathrm{C} 1-\mathrm{C} 6-\mathrm{C} 5 & -0.3(2) & & \end{array}$

Hydrogen-bond geometry $\left(A,{ }^{\circ}\right)$

\begin{tabular}{lllll}
\hline$D-\mathrm{H} \cdots A$ & $D-\mathrm{H}$ & $\mathrm{H} \cdots A$ & $D \cdots A$ & $D-\mathrm{H} \cdots A$ \\
\hline $\mathrm{C} 9-\mathrm{H} 9 B^{\cdots} \cdots 4^{\mathrm{i}}$ & 0.98 & 2.40 & $3.375(2)$ & 174 \\
$\mathrm{C} 10-\mathrm{H} 10 B \cdots \mathrm{O} 6^{\mathrm{ii}}$ & 0.98 & 2.51 & $3.472(2)$ & 169 \\
\hline
\end{tabular}

Symmetry codes: (i) $-x, y-1 / 2,-z+1 / 2$; (ii) $-x+1,-y+1,-z+2$. 